\title{
The Wu Xing Theory and Homeostatic Interaction of Organs
}

\author{
Yevgeny V. Albegov, Dmitry V. Butenko, Lyudmila N. Butenko \\ CAD/CAE Systems Department; Volgograd State Technical University, Volgograd, Russia \\ E-mail:albegov05@mail.ru,butenko@vstu.ru \\ Received June 21, 2010; revised August 9, 2010; accepted August 13, 2010
}

\begin{abstract}
In this paper the making of homeostatic models of the bioenergetic informational acupunctural human's system of the "Pentacube" and the "Hexagon" according to the Wu Xing rules and energy shifts dynamics is considering. The making of the meridional homeostatic model of the bioenergetic informational acupunctural human's system based on the existing knowledges about the human body meridians is considering. Preference to choice of the homeostatic functional models of the human body organs energies interaction is substantiated. The meridional control submodel of homeostatic system is defined and constructed. The description of control submodel, perspectives of application and development are represented.
\end{abstract}

Keywords: System Stability, Homeostat, Homeostatic Model, "Pentacube", "Hexagon”, Meridional Homeostatic Model, Control Submodel, "Merkabah"

\section{Introduction}

The fundamental issues of any system are the problems of stability. In this paper various types of stability and models, this stability is achieved with, are considering. As a prototype, we consider how to reach stability in complex systems, which are biological organisms.

For biosystems the stability issues is the problem of homeostasis [1]. As an example of this system class stability, we selected the human organism and examined it based on the five-membered cycle of the Wu Xing which reflects the interaction of human organs groups.

In the process of studying of organs rhythm activity of the Yin-Yang cycles, we came to the conclusion that the interaction is homeostatic in nature. Then based on this conclusion we constructed the functional homeostatic "Pentacube" model. Next, on the basis of data available to us, the sixth group of organs of the "Fire' " element was extracted. As a result we increased the order of the system stability transforming the "Pentacube" into the "Hexagon" model.

The next step of the model development was the extraction of regulating channels of processes and their switching centers in the homeostatic relationships. They are represented by the meridians of the human body, encephalon and spinal cord.

Based on the information about the levels of the system organization processes, we have attempted to identify the implicit control structure of the meridional homeostatic system. On the basis of representation of two-dimensional discrete set of stars we have concluded that the control submodel is the "Merkabah" system.

The stellated tetrahedron is an invariant of the octahedron, and hence, it is quite a stable structure in different coordinate systems. The "Merkabah" submodel also proves the correctness of the hexagonal homeostatic model and the meridional homeostatic model: a stable system of biological type can be controlled only by a stable internal structure with the same stability level. In systems of the pentagonal type the correct stable internal model is not observed.

Thus, the stability of the human body functioning can be explained in terms of homeostatic nets.

\section{Making of the Homeostatic Models}

\subsection{Making of the "Pentacube"}

According to the Wu Xing theory, the pentagons of human organs Yang and Yin have two circular cycles. One is clockwise and stimulating in nature, the other is anticlockwise and depressive [2]. The pentagons' stimulating and depressive processes can be graphically represented as a pentagram (a five-pointed star). This 
may be due to the interaction between two poles of homeostats. Besides, if you combine the Yin and Yang organs relating to the same element according to the Wu Xing theory, you can get a number of Yin-Yang homeostats. The homeostatic nature of this interaction is confirmed by the fact that organs interactions in these homeostats are synchronized. For example, in homeostat of "Metal" element (lungs-large intestine) lungs become more active from 3 till 5 in the morning, and large intestine from 5 till 7 in the morning; in homeostat of "Water" element (kidneys - urinary bladder) urinary bladder becomes more active from 15 till 17 in the afternoon, and kidneys from 17 till 19 in the afternoon; in homeostat of "Wood" element (liver-gall bladder) gall bladder becomes more active from 23 till 1 in the night, and liver from 1 till 3 in the night; in the homeostat of "Fire" element (small intestine-heart), the heart becomes more active from 11 till 13 in the afternoon, and small intestine from 13 till 15 in the afternoon; in the homeostat of "Earth" element (spleen/pancreas-stomach) stomach becomes more active from 7 till 9 in the morning, and spleen/pancreas from 9 till 11 in the morning. So, connecting all 5 known organs of Yin and Yang horizontally and vertically, we get the homeostatic functional "Pentacube", in the vertexes of which there are human organs, and the edges are homeostatic connections between organs (Figure 1).

The direction of arrows on the figures of homeostatic models indicate "generating" (the green one) and "overcoming" (the black one) processes accepted and thoroughly described in the Wu Xing theory.

In particular, alongside with "Pentacube" model one obtains a partial fractal model of the relationships hierarchy of homeostats. At the same time, the chain of "the conglutinated" homeostats represents the homeostat of higher organizational level. The new homeostat processes information in the same manner as the component homeostats do but at qualitatively superior level [3].

\subsection{Making of the "Hexagon"}

First of all, the premises for the existence of sixth group

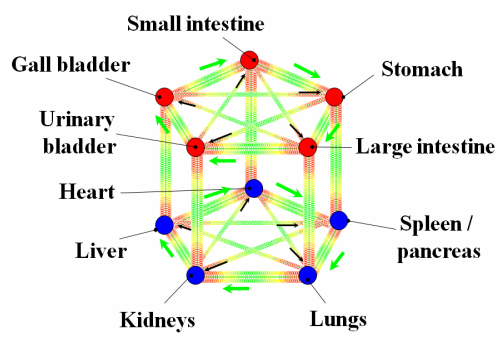

Figure 1. Homeostatic “Pentacube” of organs were made many hundreds years ago by ancient Chinese scholars. In parallel with the system of five Zang-Fu, they created a system of circulation of the twelve meridians (Jing-Luo), the imaginary vessel, which circulates Qi energy, usually called "vital force". After developing the system of meridians, the ancient Chinese scholars associated each meridian with a specific internal organ. Meanwhile, onto the 12 meridians only 10 of the internal organs were assigned. So, the question if two more internal organs existed arose. For the additional Zang organ, the so-called "Master of the Heart" (Xin Zhu) also known as pericardium was selected. Onto the place of the sixth Fu organ, the "super-organ" was selected. It is known as San Jiao or triple heater. This organ of Three Jiaos is involved in metabolic processes of the organism. Currently, one can come across the fact that in addition to five-membered ring in the Wu Xing cycle, there is another equal "member". The "Fire" element is dual and contains 4 elements (two Zang organs and two Fu organs) in that representation.

Secondly, although the element of "Fire" fills the human body with vital energy, the physical-chemical mechanism of oxidation ("internal warming") in the "Fire" of heart-small intestine and in the "Fire" of pericardium - triple heater is different.

Thirdly, we turn to the stability of systems built on polygons. Hexagon has a higher symmetry axis L6 against the pentagonal L5. Structures of solids, characterized by the fifth-order axis of symmetry of the prohibited in classical crystallography, occur rarely in nature and have a prefix "quasi-". The very nature aspires to create objects of different complexity levels in the hexagonal structures. This property is manifested both at the macrocosm and microcosm levels.

Fourthly, the interaction rhythm (by analogy with the previously described homeostats) of organs such as pericardium - triple heater will also be homeostatic in nature: the pericardium becomes more active from 19 to 21 in the evening, and triple heater from 21 till 23 in the evening.

And fifth, in a model of five-membered cycle in pathological states of excess or lack of Qi energy in the organs, logical rhythmic of activity has a probabilistic nature, and points to an extreme degree of degeneration of the pentagonal system. In the model of six-membered cycle this uncertainty states is completely eliminated.

The ideas described above enable us to draw a conclusion that the sixth group of organs exists: triple heaterpericardium, relating to the element "Fire' ". The processes of stimulating and suppression and, accordingly, the homeostatic interactions of organs in sexangles of Yin and Yang of the homeostatic functional "Hexagon" are the same as the processes between the organs in the pentagons of "Pentacube" (Figure 2). 


\section{Making of the Meridional Homeostatic Model}

Due to the fact, there are regulators in the compound of homeostatic bioenergetic system, then we suggest, that as channels of regulating the standard meridians are used, which have projections on the surfaces of human body. It is known, that besides 12 standard meridians, related to the definite organs of the human body, there are other subsystems of meridians, performing the functions of additional direct and feedback connections ("miraculous" meridians, tendinomuscular meridians, unpaired meridians, collaterals and secondary meridians) [4]. To switching centers, transmitting control action into the unpaired posteromedian and unpaired anteromedian pretend, to our mind, spinal cord for Yin organs and encephalon for Yang organs. Chief-regulator likely to represent consis- tent beginning of nature, pacing the works of homeostats of organs. Since inner homeostatic interactions between the organs in pentagon of organs are described as a five- point star (a star as a planar geometric figure is meant) and are exhaustive, so in sexangle of organs the inner homeostatic interactions will be described as a six-point star/hexagram, representing in form the projection of stellated trigonal bipyramid on the plane, and will be also exhaustive $[5,6]$. Consequently the conclusion can be made about the presence of homeostatic connections between the homeostat of "Wood" element (liver-gall bladder) and homeostat of "Fire' " element (pericardium-triple heater), and between the homeostat of the "Fire" element (small intestine-heart) and the homeostat of "Earth" element (spleen/pancreas - stomach) in homeostatic "Hexagon" of the human body organs (Figure 3).

The given model of interaction of human organs energies represents a hierarchy meridional and homeostatic net, where the meridional net represents a tracking system with feedback connection on the wholeness of homeostatic net system.

The preference of choosing forms of homeostatic models

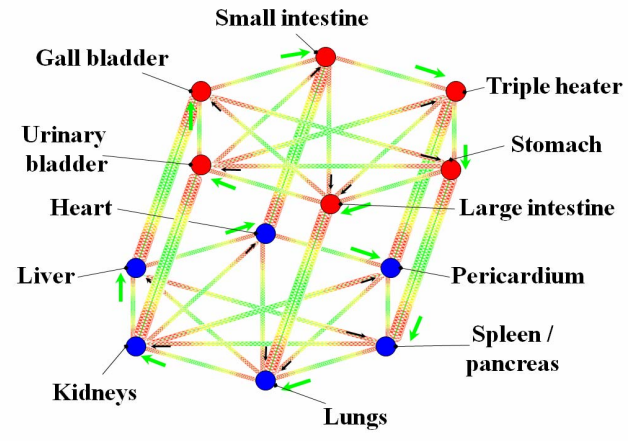

Figure 2. Homeostatic “Hexagon”. is based on "gold" polygons and polyhedrons since the structures having in its basis correct n-angles have the maximal degree of stability, information, self- organizing and a harmonicity, whether there are creations of civilizations (the Egyptian pyramids, protective forts, calendars of ancient civilizations, the theory of "civilizing hexagon", models of location of cities, models of classification of smells) or the very nature (structure of che- mical substances (fullerenes, frame structures), of a atomic nucleus, DNA chain, a crystal lattice and nanostructures) [7]. "The golden section" and corresponding "golden" properties of edges of functional homeostatic models are based on concepts of space of variability of natural processes and homeostat (elementary homeostatic system) [8].

\section{Making of the Meridional Submodel "Merkabah"}

Proceeding from judgment that organizers and regulators of local processes in the control space of the lower level of system of correct polygons are stars (connected and not connected), it is possible to make a conclusion, that in homeostatic "Hexagon" (a polyhedron - a prism) of the meridional homeostatic system of energies interaction of human body organs such organizer-regulator of processes is a star-shaped polyhedron-Merkabah or a stellated tetrahedron.

Merkabah as a member of a row of Platonic solids (octahedron is an invariant to stellated trigonal bipyramid) can model dualism. Merkabah as a process is the integral stage of biosystem genesis, namely biological-energy evolution of a cell: at the third stage of the ovicell division eight cells form a stellated tetrahedron and they are absolutely identical to each other [7]. Static cognitive model of interaction a stellated tetrahedron reflects the basic

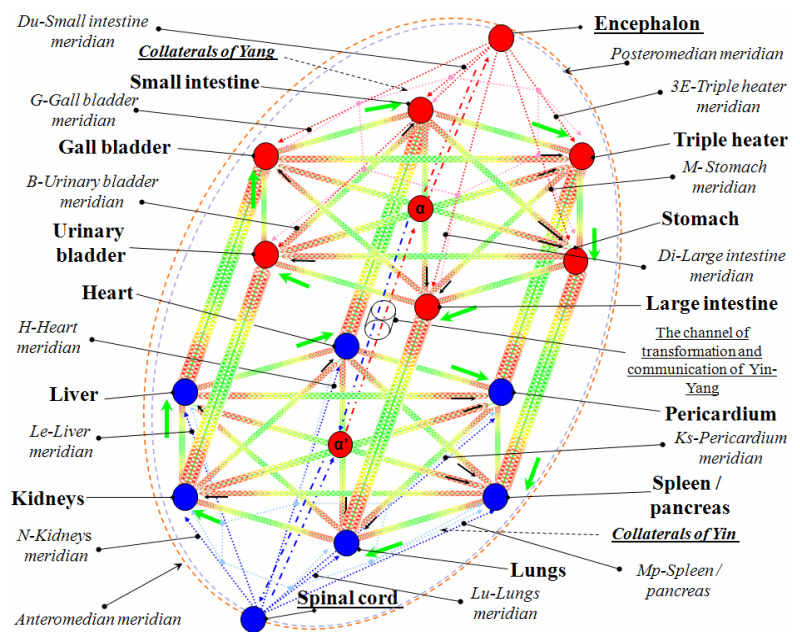

Figure 3. Meridional homeostatic model. 
rules of constructing of meridional biosystem and the technology of forming homeostatic connections as complete units. Knots in "Merkabah" system represent a brain as the switching centers of a human body. 4 knots of Yang-tetrahedron are the encephalon, and 4 knots of Yin-tetrahedron - the spinal cord. And as the switching centers encephalon and the spinal cord will be absolutely identical to character of controlling.

The key rule of constructing consists of the following: the meridian represents not unidirectional structural unit (brain - body), but a contour (brain - organ - brain), or a construction of the tunnel type with "two-way traffic". The technology of formation of homeostatic units is obviously displayed on a stellated trigonal bipyramid: homeostatic couple of Yin-Yang organs is formed owing to a crossing of meridians of the corresponding Yin-Yang organs. We can notice that such approach does not contradict, namely proves the correctness of meridional homeostatic model. Having connected homeostatic couples of Yin-Yang organs by a broken line in view of their timely activity, we receive a flat figure hexanema, determining a timely way [9] (Figure 4).

Proceeding the meridians in view of timely activity both in a stellated tetrahedron as a whole, and in Yin and Yang tetrahedrons, and consequently also a process of meridional energy circulation (signals), on the given cognitive models occurs at first clockwise, then changes its direction on counter, i.e. on an endless helix, and on a plane looks like an infinity sign.

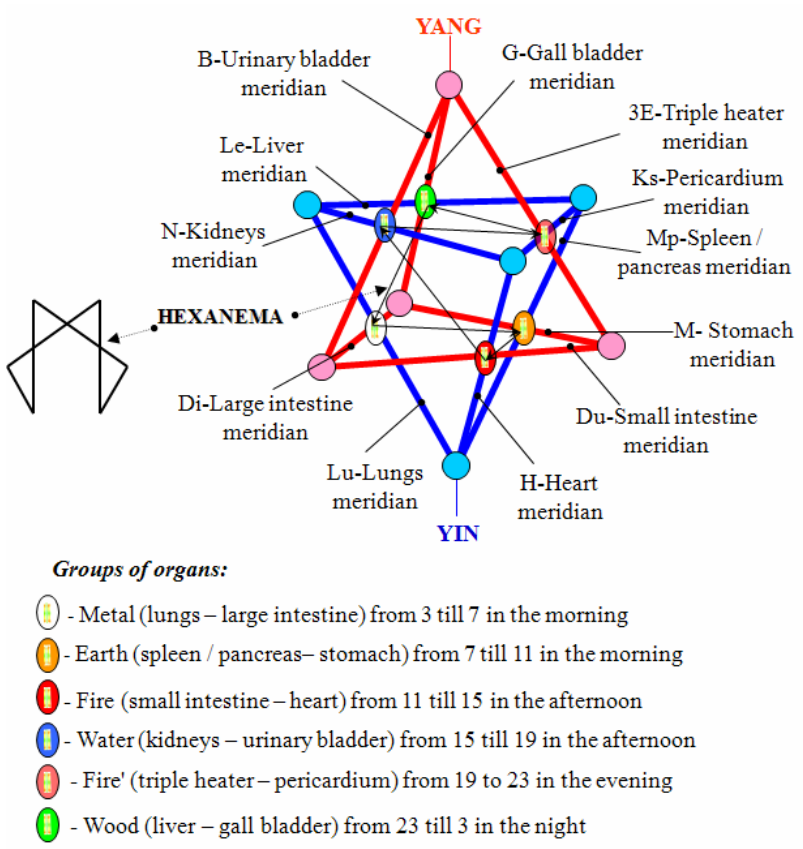

Figure 4. Meridional submodel “Merkabah”.

\section{Conclusions}

The system approach to the construction of functional submodel allows to observe all scope of relations of "Merkabah" elements and connections between them and to approach to understanding of dialectic "plan" of the nature in organizing and controlling the processes, to see dynamics in statics. From the point of view of the applied component of the research, the given control submodel allows to understand the essence of pathological processes in a human body, as infringement of energy flow (transferring signals) in meridional system with the further disbalance of rhythms of homeostats and displace ment of homeostatic energy from optimum areas to the risky areas. The same comprehension of occurrence of disease states in turn, on the basis of knowledge of traditional Chinese medicine, will lead to forming and using of algorithms of updating of biosystem and taking out from pathological conditions by methods of not classical medicine, but phytotherapy or acupuncture. We'd like to note that "Merkabah" as conceptual model of relations of complicated compound elements, has all preconditions to deep system complication (by all aspects of the given concept), owing to the analysis and decomposition of components.

\section{References}

[1] A. G. Teslinov, "Development of Control Systems: Methodology and Conceptual Structures," Globus, Moscow, 1998.

[2] Wu Xing, 2005. http://en.wikipedia.org/wiki/Wu_Xing

[3] A. M. Stepanov, "The Fundamental Principle of the Medical Homeostatics," MODEC, Voronezh, 1994.

[4] G. Luvsan, "Traditional and Modern Aspects of Oriental Acupuncture," Nauka, Moscow, 1991.

[5] The Star (geometry), 2008. http://ru.wikipedia.org/wiki/ Звезда_(геометрия)

[6] The Bipyramid, 2008. http://ru.wikipedia.org/wiki/Бипирамида

[7] A. Stahov and A. Sluchenkova and I. Scherbakov, "The Da Vinci code and Fibonacci series," Piter, SPb, 2007.

[8] Yu. M. Gorsky and A. M. Stepanov and A. G. Teslinov, "Homeostatic: Harmony in Game of Contradiction," Reprotsentr A1, Irkutsk 1998.

[9] V. Eremeev, "A Diagram of Anthropocosmos," ASM, Moskva, 1994. 